\title{
Theoretical evolution of Rossby number for solar analog stars
}

\author{
Matthieu Castro, Tharcísyo Duarte and José Dias do Nascimento Jr. \\ Departamento de Física Teórica e Experimental (DFTE), Universidade Federal do Rio Grande \\ do Norte (UFRN), Campus Universitário Lagoa Nova, CEP: 59078-970, Natal (RN), Brazil \\ email: mcastro@dfte.ufrn.br
}

\begin{abstract}
Magnetic fields of late-type stars are presumably generated by a dynamo mechanism at the interface layer between the radiative interior and the outer convective zone. The Rossby number, which is related to the dynamo process, shows an observational correlation with activity. It represents the ratio between the rotation period of the star and the local convective turnover time. The former is well determined from observations but the latter is estimated by an empirical iterated function depending on the color index $(B-V)$ and the mixing-length parameter. We computed the theoretical Rossby number of stellar models with the TGEC code, and analyze its evolution with time during the main sequence. We estimated a function for the local convective turnover time corresponding to a mixing-length parameter inferred from a solar model, and compare our results to the estimated Rossby number of 33 solar analogs and twins, observed with the spectropolarimeters ESPaDOnS@CFHT and Narval@LBT.
\end{abstract}

Keywords. solar analogs, magnetic field, Rossby number

\section{Evolutionary models}

Stellar evolution calculations were computed with the Toulouse-Geneva stellar evolution code TGEC. Details of the input physics used in our models can be found in do Nascimento et al. (2013). The evolution of the angular momentum is calculed with the Kawaler (1988) law, and for the initial rotation rates, we adopted the relation (3) in Landin et al. (2010). The calibration method of the models is based on the Richard et al. (2004) prescription: a solar model is calibrated to match the observed solar radius and luminosity at the solar age. The parameter $K$ of the angular momentum evolution law is adjusted to give the solar rotation period $\left(P_{\text {rot }}=27.1 \mathrm{~d}\right)$ at the solar age. Evolutionary models of masses $0.81,0.85,0.90,0.95,1.00,1.05$, and $1.10 \mathrm{M}_{\odot}$ were calculated from the ZAMS to the top of the RGB. The input parameters for the other masses are the same as for the $1.00 \mathrm{M}_{\odot}$ model.

\section{Stars sample}

To compare with our models, we use a sample of 33 sun-like stars observed with the spectropolarimeters ESPaDOnS at the Canada-France-Hawaii Telescope (Mauna Kea, USA) and NARVAL at the Télescope Bernard Lyot (Pic du Midi, France). Longitudinal magnetic field of the stars were determined with the technique of Least-SquareDeconvolution (LSD, Donati et al. 1997). Rotation periods of the stars, determined from chromospheric activity, were found in the literature (Noyes et al. 1984, Wright et al. 2004, Lovis et al. 2011). 

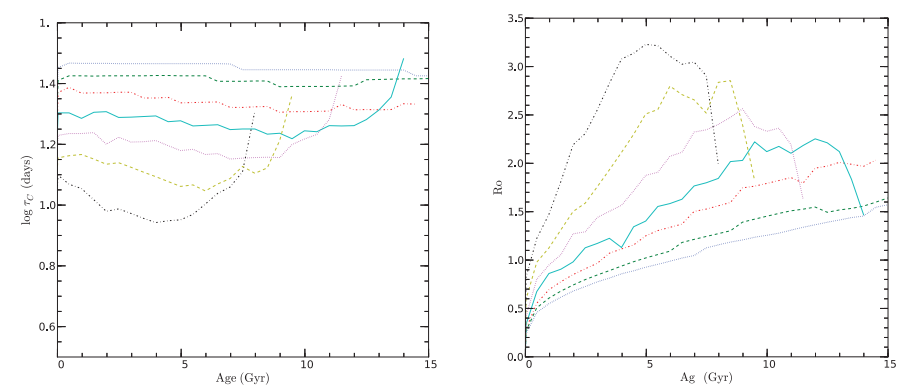

Figure 1. $\log \tau_{\mathrm{C}}$ (left) and Rossby number Ro (right) as a function of age for our models of 0.81 (dotted), 0.85 (dashed), 0.90 (dot-dashed), 0.95 (continuous), 1.00 (thick dotted), 1.05 (thick dashed), and 1.10 (thick dot-dashed) $\mathrm{M}_{\odot}$.
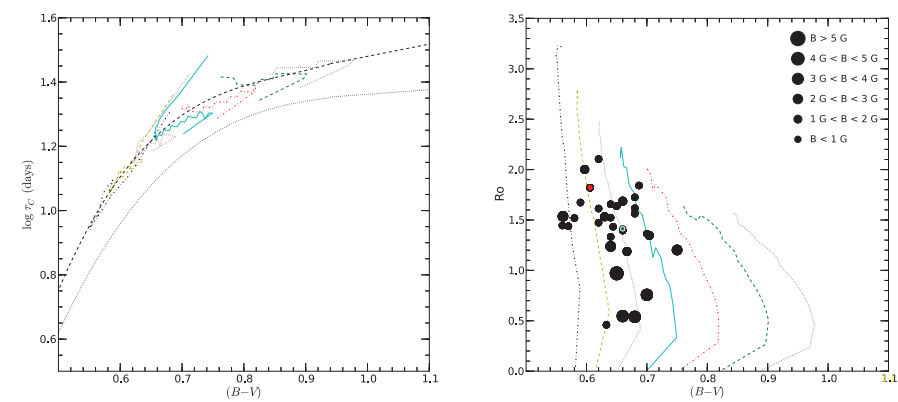

Figure 2. Left: $\log \tau_{\mathrm{c}}$ as a function of $(B-V)$. The dashed line is the polynomial regression of the models tracks. The dotted line is the polynomial regression found by Noyes et al. (1984). Right: Rossby number $R o$ as a function of $(B-V)$ for our models limited to the main sequence. Filled black circles are observed stars, the size depends on the strength of the magnetic field.

\section{Rossby number}

For all the models, we calculated the Rossby number $R o=P_{\text {rot }} / \tau_{\mathrm{c}}$ where $P_{\text {rot }}$ is the rotation period, and $\tau_{\mathrm{c}}$ the local convective turnover time, calculated at a distance of one pressure height scale $H_{\mathrm{P}}$ above the base of the convective zone. Its value is computed through the equation $\tau_{\mathrm{c}}=\alpha H_{\mathrm{P}} / v$, where $v$ is the convective velocity. In Fig. 1 , we plot the evolution of $\log \tau_{\mathrm{c}}$ (left panel) and $R o$ (right panel) as a function of the stellar age.

In the left panel of Fig. 2, we show the $\log \tau_{\mathrm{c}}$ of our models as a function of $(B-V)$. A cubic fit through these curves (dashed line) defines an empirical function $\log \tau_{\mathrm{C}}(B-V)$, given by: $\log \tau_{\mathrm{c}}=-5.468+22.810(B-V)-25.637(B-V)^{2}+9.796(B-V)^{3}$. From this equation, we determine the $\tau_{\mathrm{c}}$ of the stars of our sample, and then a Rossby number $R o$. In the right panel of Fig. 2 we plot the Rossby number as a function of $(B-V)$ for our models and for the stars of our sample, determined from the observed rotation period and the convective turnover time inferred from the above equation.

\section{References}

do Nascimento, J.-D., da Costa, J. S., \& Castro, M. 2013, A\& A, 548, L1

Donati, J.-F., Semel, M., Carter, B. D. et al. 1997, MNRAS, 291, 658

Kawaler, S. D. 1988, ApJ, 333, 236

Landin, N. R., Mendes, L. T. S., \& Vaz, L. P. R. 2010, A\&A, 510, A46

Lovis, C., Dumusque, X., Santos, N. C. et al. 2011, eprint arXiv:1107.5325

Noyes, R. W., Hartmann, S. W., Baliunas, S. et al. 1984, ApJ, 279, 763

Richard, O., Thado, S., \& Vauclair, S. 2004, SoPh, 220, 243

Wright, J. T., Marcy, G. W., Paul Butler, R., \& Vogt, S. S. 2004, ApJS, 152, 261 